\title{
Exploring perspectives, preferences and needs of a telemonitoring program for women at high risk for preeclampsia in a tertiary health facility of Karachi: a qualitative study protocol
}

Anam Feroz ${ }^{1 *}$, Sarah Saleem ${ }^{1}$ and Emily Seto ${ }^{2,3}$

\begin{abstract}
Background: In Pakistan, deaths from preeclampsia/eclampsia (PE/E) represent one-third of maternal deaths reported at tertiary care hospitals. To reduce the morbidity and mortality associated with PE/E, an accessible strategy is to support pregnant women at high risk for preeclampsia (HRPE) by closely monitoring their blood pressures at home (i.e., telemonitoring) for the earliest signs of preeclampsia. This could lead to the earliest possible detection of high blood pressure, resulting in early intervention such as through medications, hospitalization, or delivery of the baby. The study aims to explore the perspectives, preferences and needs of telemonitoring (TM) for pregnant women at HRPE in Karachi, to inform future implementation strategies.

Methods: The study will employ an exploratory qualitative research design. The study will be conducted at the Jinnah Postgraduate Medical Centre (JPMC) hospital and Aga Khan University Hospital (AKUH) in Karachi, Sindh, Pakistan. Data will be collected through key-informant interviews (KIIs) and in-depth patient interviews (IDPIs). IDPIs will be conducted with the pregnant women at HRPE who are visiting the out-patient department/ antenatal clinics of JPMC hospital for antenatal check-ups and immunizations. Klls will be conducted with the obstetricians, Maternal, neonatal and child health (MNCH) specialists and health care providers at JPMC, as well as TM experts from Karachi. Study data will be analyzed through conventional content analysis. Interviews are anticipated to begin in April 2020 and to be completed during the summer of 2020.

Discussion: This is the first study to explore the use of TM program for pregnant women at HRPE in a tertiary health facility in Karachi. The research will help explore perceived benefits associated with the use of a TM program alongside potential facilitators and barriers that may help inform the future implementation of a TM program for pregnant women at HRPE in Karachi.
\end{abstract}

Keywords: Pregnant women, High risk for preeclampsia, Telemonitoring, Home blood pressure monitoring, Qualitative study

\footnotetext{
* Correspondence: anam.sahyl@gmail.com

'Department of Community Health Sciences, The Aga Khan University,

Stadium Road, PO Box 3500, Karachi 74800, Pakistan

Full list of author information is available at the end of the article
}

(c) The Author(s). 2020 Open Access This article is licensed under a Creative Commons Attribution 4.0 International License, which permits use, sharing, adaptation, distribution and reproduction in any medium or format, as long as you give appropriate credit to the original author(s) and the source, provide a link to the Creative Commons licence, and indicate if changes were made. The images or other third party material in this article are included in the article's Creative Commons licence, unless indicated otherwise in a credit line to the material. If material is not included in the article's Creative Commons licence and your intended use is not permitted by statutory regulation or exceeds the permitted use, you will need to obtain permission directly from the copyright holder. To view a copy of this licence, visit http://creativecommons.org/licenses/by/4.0/. The Creative Commons Public Domain Dedication waiver (http://creativecommons.org/publicdomain/zero/1.0/) applies to the data made available in this article, unless otherwise stated in a credit line to the data. 


\section{Plain English summary}

Preeclampsia is a disorder of pregnancy that is characterized by high blood pressure and protein in the urine after 20 weeks of pregnancy. Maternal and neonatal deaths from preeclampsia results from a lack of early identification of women at risk for preeclampsia, transportation difficulties to treatment centers, sub-optimal patient education on this topic, and inability to detect signs of imminent danger to the pregnant individual and baby. Telemonitoring is a prevention strategy where pregnant individuals take blood pressure measurements at home through home blood pressure machines and these readings are sent to their healthcare providers. It can enable the earliest detection of high blood pressure, resulting in early intervention such as, medications, management hospitalization, or delivery of the baby. The TM platform includes a smartphone app that automatically transfers blood pressure readings wirelessly from a blood pressure home monitor and sends automated alerts to providers and self-care instructions to the patient based on their readings. The study aims to explore perspectives of health care experts and pregnant women on the use of telemonitoring for pregnant women at HRPE in Karachi. The findings will provide a better understanding of study participants' attitudes and readiness towards the use of a TM program that is intended to be implemented at JPMC.

\section{Background}

Preeclampsia is a disorder of pregnancy that is characterized by high blood pressure and protein in the urine after 20 weeks of pregnancy. It is associated with high maternal mortality, preterm birth, pregnancy loss, and stillbirth [1]. Women with preeclampsia are at increased risk for stroke and damage to vital organs such as kidneys, liver, and brain. In severe cases, preeclampsia can lead to eclampsia which is the presence of seizures [1]. This condition could also lead to the separation of the placenta from the uterus (referred to as placental abruption) [1]. Infants born preterm due to preeclampsia are at higher risk of some long-term health issues including learning disorders, cerebral palsy, epilepsy, deafness, and blindness [1, 2]. Hypertensive disorders of pregnancy contribute to $14 \%$ of all maternal deaths, the majority of which occur in low- and middle-income countries [3].

Pakistan has one of the highest maternal mortality ratios worldwide at 178/100,000 live births [4, 5] and neonatal mortality ratios at $42 / 1000$ live births [6]. Eclampsia accounts for more than $12 \%$ of direct maternal deaths [7]. Deaths from preeclampsia/eclampsia represent one-third of maternal deaths reported at tertiary care hospitals [8]. Maternal and neonatal morbidity and mortality from preeclampsia results from a lack of early identification of women at risk for preeclampsia, transportation difficulties to treatment centers, sub-optimal patient education on this topic, and inability to detect signs of imminent danger to the pregnant individual and baby [8].

To meet the targets of the United Nations' Sustainable Development Goal 3 by 2030 (maternal mortality ratio < $70 / 100,000$ live births; neonatal mortality ratio $<=12 / 1000$ live births) [9], strategies are required to decrease the rate of mortality from preeclampsia/eclampsia. An accessible strategy to meet this goal is to support pregnant women at HRPE by closely monitoring their blood pressure at home (i.e. TM) in order to detect the earliest signs of preeclampsia. TM is a prevention strategy where pregnant individuals take blood pressure measurements at home through home blood pressure machines and these readings are sent to their healthcare providers. Automated clinical alerts can also be generated based on the patient's readings, as well as self-care messages for the patient. TM can enable the earliest detection of high blood pressure, resulting in early intervention such as, medications, management hospitalization, or delivery of the baby $[10,11]$.

Several retrospective and prospective studies have recommended the use of home blood pressure monitoring (HBPM) in pregnancy because of its potential benefits including fewer hospital visits, better blood pressure control, and cost savings [12-15]. A scoping review conducted in 2019 found 16 unique TM interventions from 20 studies [12, 14, 16-33] for patients at high-risk for hypertensive disorders of pregnancy, but these studies have not translated to sustained programs [34]. The scoping review concluded that TM for hypertension during pregnancy was feasible, convenient, and cost-effective. Most TM interventions have been implemented in high-income countries including UK, USA, and Belgium. There is little evidence available on the use of a TM platform for pregnant women in LMICs. Thus, the feasibility and effectiveness of TM interventions to support pregnant women at HRPE in a low-middle-income setting may be different due to differing patient demographics, healthcare services available, cultural considerations, budgetary constraints and clinical workflows. Implementing a TM program for pregnant women at HRPE in Karachi, Pakistan would require a thorough understanding of perceived benefits associated with the use of a TM program alongside potential facilitators and barriers that may help inform the future implementation of such a TM program in a tertiary health facility of Karachi. In addition, exploring the readiness of the deployment site towards the use of a TM program for pregnant women at HRPE, is particularly essential to ensure engagement of the deployment site during the implementation phase. Prior to implementing a similar TM program in Karachi, Pakistan, it is crucially important to explore the perspectives and readiness of the healthcare professionals' and end users (pregnant women at HRPE) regarding the use of TM for preeclampsia. 


\section{Study aim \& objectives}

The aim of this study is to explore the perspectives, preferences and needs of TM for pregnant women at HRPE in Karachi, to inform a potential future implementation. In particular, a smartphone-based TM platform developed in Canada is being considered as a possible system for TM of pregnant women at HRPE in Karachi [35-44]. The TM platform includes a smartphone app that automatically transfers blood pressure readings wirelessly from a blood pressure home monitor and sends automated alerts to providers and self-care instructions to the patient based on their readings. The specific objectives of this study are:

1. To explore pregnant women's perspective, preferences and needs of TM, and in particular smartphone-based TM, for pregnant women at HRPE in Karachi.

2. To explore the perspectives of obstetricians, healthcare providers (doctors, nurses and midwives), Maternal, neonatal and child health $(\mathrm{MNCH})$ specialists, and TM experts on the use of TM for pregnant women at HRPE in Karachi.

\section{Methods}

\section{Study design}

This formative research will employ an exploratory qualitative research design using semi-structured interviews and a purposive sampling approach. The data collection methods for this formative research will involve key-informant interviews (KIIs) and in-depth patient interviews (IDPIs).

\section{Study setting}

The study will be conducted at Jinnah Postgraduate Medical Centre (JPMC) hospital and Aga Khan University Hospital (AKUH) Karachi, Sindh, Pakistan. JPMC serves over one million patients of low-socio-economic status per year from Karachi, Interior Sindh, Baluchistan and other areas. Each year, approximately 60,000 patients are admitted, with 30,000 surgeries and 15,000 deliveries, all free of charge. JPMC is a 1650-bed tertiary care public sector hospital with over 31 departments [45]. Pregnant women at HRPE will be identified from the out-patient department (OPD) area where women wait for several hours to be seen by their healthcare providers.

AKUH was established in 1985 as one of the principal teaching sites for nurses and doctors from the university's School of Nursing and Midwifery and the School of Medicine. AKUH's multidisciplinary approach to diagnosis and care ensures a continuum of safe and high-quality care for patients, with all services under one roof. Key informants' such as obstetricians, gynecologists, and few TM experts will be identified from relevant departments of Aga Khan University.

\section{Data collection methods Key-informant interviews (KIls)}

We will invite 'key informants' such as obstetricians, healthcare providers, $\mathrm{MNCH}$ specialists, and TM experts to understand their perspectives, preferences and needs regarding use of TM for pregnant women at HRPE.

The KIs will be identified from AKUH, JPMC, Nongovernmental organizations (NGOs) and the Aga Khan Development Network Digital Health Resource Center (AKDNdHRC) and other relevant institutions. A few KIIs with obstetricians and healthcare providers (doctors, nurses and midwives at JPMC) will be arranged at JPMC. A few obstetricians and MNCH specialists will be identified from AKUH and NGOs. Experts in the field of TM will be identified and interviewed from AKUH and the AKDNdHRC to understand the technological feasibility of TM intervention in Karachi, Pakistan. KIs will be invited to participate in the qualitative study via email. KIs will be requested to sign consent forms (Additional file 1) before the interview begins, in which they will agree that the interview can be audio-recorded and written notes can be taken by a note-taker to record any relevant observations. It is anticipated that the interviews will be conducted in the languages of Urdu or Sindhi. Initially, the KII will involve discussion around causes of $\mathrm{PE}$ and routine obstetric care for PE. Later, the discussion will move towards exploring participant views towards use of TM for pregnant women at HRPE. Finally, the interview will explore perceived facilitators and barriers for implementation of TM, including smartphonebased TM. We anticipate that 13-15 participants will be recruited for KIIs, but additional interviews will be conducted until data saturation. Each KII will take approximately between 30 and $45 \mathrm{~min}$.

\section{In-depth patient interviews (IDPIs)}

IDPIs will be conducted with pregnant women at HRPE who are visiting the OPD/antenatal clinics of JPMC hospital for antenatal check-ups and immunizations. Pregnant individuals at HRPE will be identified using National Institute for Health and Care Excellence (NICE) guidelines. Considering the cultural and ethical sensitivity of preeclampsia, the research will not have interviews with high risk pregnant women who are admitted at the emergency department and those who are inpatients. Pregnant women at HRPE will be identified from the OPD area where women wait for several hours to be seen by their healthcare providers. Recruiting pregnant women at HRPE, from a busy OPD area would be challenging and therefore healthcare providers at JPMC involved in antenatal clinic such as midwives, nurses and 
technicians, will be requested to support identification of pregnant individuals at HRPE. The study coordinator will then explain the study objectives and procedures to eligible pregnant individuals at HRPE and will obtain written consent for participation in the study (Additional file 1). If the participant is unable to read the consent form, then the study coordinator will explain the consent form verbally in their local language. Participants who are unable to write their names will be asked to provide a thumbprint to mark their consent to participate. Women who provide consent will be asked to move to a separate private room near the OPD area of JPMC, to conduct IDPIs with high-risk pregnant women without any disruption. Caregivers would be able to accompany the patient during the interviews if the patient prefers. The interview will be conducted by a trained researcher (study Principal Investigator) who is experienced in conducting qualitative research. Participants will be assured that their anonymity will be maintained, and no identifying information will be included on the transcripts. The interview will involve a general discussion about healthy pregnancy related to being HRPE, knowledge about preeclampsia (PE), causes of PE, perceptions towards use of TM for pregnant women at HRPE, perceived benefits of TM, potential limitations or concerns related to $\mathrm{TM}$ for $\mathrm{PE}$, and feasibility of smartphone-based TM. We anticipate that 10-15 IDPIs will be conducted with pregnant women at HRPE (Table 1). However, additional IDPIs will be conducted until data saturation. Each IDI will approximately take between 30 and $45 \mathrm{~min}$.

\section{Study participants}

Purposive sampling technique will be used to identify and recruit KIs and IDPIs participants for the study. Detailed eligibility criteria are given below:

\section{Eligibility criteria}

Inclusion criteria Pregnant Individuals at HRPE (IDPIs)
- Based on the National Institute for Health and Care Excellence (NICE) guidelines, pregnant individuals at HRPE will be defined as those who have one high risk factor or more than one moderate risk factor for preeclampsia [46].

High risk factors include:

- Hypertensive disease in a previous pregnancy

- Chronic kidney disease

- Autoimmune disease, such as systemic lupus erythematosus or antiphospholipid syndrome

- Type 1 or type 2 diabetes

- Chronic hypertension

Moderate risk factors include:

- First pregnancy

- Age 40 years or older

- Pregnancy interval of more than 10 years

- Body mass index (bmi) of $35 \mathrm{~kg} / \mathrm{m} 2$ or more at first visit

- Family history of preeclampsia

- Multi-fetal pregnancy

- Pregnant individuals at HRPE, who are willing to give consent to participate in the study and are able to speak either Urdu or Sindhi language

\section{Key-informants}

- Healthcare professionals who are directly or indirectly involved in the care of pregnant individuals at HRPE such as, obstetricians, healthcare providers (doctors, nurses and midwives at JPMC), MNCH specialists, and TM experts

\section{Exclusion criteria}

- The study will not include women who are newly diagnosed as HRPE (i.e., recruiting during follow-up visits at least 1 week after being diagnosed as HRPE), as they may not have had time to experience or reflect on the associated challenges.

Table 1 Study Participants for KIls and IDPIs

Participants for Key-informant Interviews (KIIs)

Sample

Range

Obstetricians at JPMC \& AKUH

03-05 Klls

Healthcare providers at JPMC (Doctors/ nurses/ midwives)

03-05 Klls

Maternal, neonatal and child health specialists at NGOs and AKUH

03-05 Klls

Telemonitoring experts at AKDNdHRC

03-05 Klls

Participants for In-depth Patient Interviews (IDPIs)
Sample

Range

10-15 IDIs 
- Considering the cultural and ethical sensitivity, the research will not include interviews with high risk pregnant women who are admitted to the emergency department and in-patient areas for treatment purpose.

\section{Data collection procedure}

Separate semi-structured interview guides have been developed for KIIs and IDPIs (Additional files $2 \& 3$ ). Both the interview guides will be pilot tested with a non-study sample (2 KIIs \& 2 IDPIs) who have the same characteristics as the study sample. The pilot testing will offer evidenced-base guidance to improve data collection guides [47].

To mitigate bias, the researcher will first explore patient participants' views towards the use of TM for pregnant women at HRPE, perceived benefits of TM, potential limitations or concerns related to TM for PE, and implementing TM for PE into clinical practice. Then, the researcher will show a power-point presentation to the patient participants to introduce the idea of TM for pregnant women who are at HRPE. The presentation will be made in the participant's local language (Urdu or Sindhi) and will mainly include graphics and illustrations to help ensure participants understand the concepts of TM and HBPM. The presentation will provide an overview on the disease scenario of preeclampsia, information on routine obstetric care of PE, and description on the potential future use of TM for high risk pregnant women. Later, the researcher will explore participants' perspectives, preferences and needs regarding use of a TM program for women at HRPE in Karachi. It is possible that the pregnant women at HRPE may feel anxiety from being asked questions about their experiences and knowledge of managing their HRPE condition. To address that, the investigator will explain to the participants the study objectives and answer any queries arising from the interview. At the end of the interview, a PowerPoint presentation will be given to patient participants to provide them with an understanding of preeclampsia, its consequences, and simple ways to manage the condition through regular follow-ups and blood pressure monitoring. Interviews are anticipated to begin in April 2020 and to be completed during the summer of 2020 .

\section{Data analysis}

The audio recordings from the interviews will be transcribed and will then be translated into English for conventional content analysis [48-50]. No identifying characteristic will be included in the transcriptions. The transcripts will be uploaded into NVivo 12 Plus software to enable easy and organized retrieval of data for analysis. Transcripts will be read several times to develop an interpretation of the participants' perception regarding the use of TM for pregnant women at HRPE. This will involve an iterative process where data will be coded, compared, contrasted and refined to generate emergent themes. This iterative process will involve revisiting the data or going back and forth repeatedly on the data to address the data gaps and to seek information to saturate the gaps in subsequent contacts with new research participants [51]. The transcribed text will be divided into 'meaning units' which will be later shortened and labeled with a 'code' without losing the study context. Codes will be then analyzed and grouped into similar categories. In the final step, similar categories will be assembled under sub-themes and main themes. Two independent investigators will perform the coding, and category creation, and discrepancies will be resolved to through discussion until a consensus is reached. To gain a more complete understanding of the perspectives, preferences and needs of TM for women at HRPE, the themes from the KIIs and IDPIs will be compared and contrasted.

\section{Ethical considerations}

Ethical approval for this study has been obtained from the Aga Khan University Ethical Review Committee (AKU-ERC) - [2020-2153-8519].

\section{Discussion}

This is the first study to explore the use of TM program for pregnant women at HRPE in a tertiary health facility in Karachi. This research will help explore perceived benefits associated with the use of a TM program, as well as potential facilitators and barriers that may inform the future implementation of a TM program for pregnant women at HRPE in a tertiary health facility in Karachi. Specifically, the findings of the qualitative study will provide a better understanding of study participants' attitudes and readiness towards the use of a TM program that is intended to be implemented at JPMC. This study would inform an implementation blueprint, by suggesting tailored strategies for future successful implementation of TM programs across Pakistan.

\section{Supplementary information}

Supplementary information accompanies this paper at https://doi.org/10. 1186/s12978-020-00979-8.

Additional file 1. Informed Consent.

Additional file 2. Semi-Structured Interview Guide for KIIs.

Additional file 3. Semi-Structured Interview Guide for IDPIs.

\section{Abbreviations}

HBPM: Home blood pressure monitoring; HRPE: High risk for preeclampsia; IDPIs: In-depth interviews; KIls: Key-informant interviews; MNCH: Maternal, neonatal and child health; TM: Telemonitoring; AKDNdHRC: Aga Khan Development Network Digital Health Resource Center; NGOs: Nongovernmental organizations; AKUH: Aga Khan University Hospital Karachi; JPMC : Jinnah Postgraduate Medical Centre 


\section{Acknowledgements}

Not applicable.

\section{Authors' contributions}

ES and AF conceptualized and designed the study. AF prepared the first draft of the manuscript. ES reviewed the manuscript several times and provided critical feedback. All authors (AF, ES, SS) read and approved the final version of the manuscript.

\section{Funding}

The study is funded by Aga Khan University, Faculty of Health Sciences under Seed Money Programme for Research Development.

\section{Availability of data and materials}

Materials described in this paper pertain to the study protocol only and there are no raw data reported. The datasets will be collected and analyzed and can be made available from the corresponding author on reasonable request.

Interview guides developed for this study protocol are included as Additional files 2 \& 3.

\section{Ethics approval and consent to participate}

Ethical approval for this study has been obtained from the Aga Khan University Ethical Review Committee (AKU-ERC) - [2020-2153-8519]. Participants are required to sign a consent form to indicate their willingness to participate. Voluntary participation and the right to ask any questions and to decline participation at any time will be emphasized during the data collection.

\section{Consent for publication}

Not applicable.

\section{Competing interests}

The authors declare that they have no competing interests.

\section{Author details}

'Department of Community Health Sciences, The Aga Khan University, Stadium Road, PO Box 3500, Karachi 74800, Pakistan. ${ }^{2}$ Institute of Health Policy, Management and Evaluation, Dalla Lana School of Public Health, University of Toronto, Toronto, ON, Canada. ${ }^{3}$ Centre for Global eHealth Innovation, Techna Institute, University Health Network, Toronto, ON, Canada.

Received: 5 August 2020 Accepted: 9 August 2020

\section{Published online: 15 September 2020}

\section{References}

1. National Institute of Child Health and Human Development. (2017) Preeclampsia and Eclampsia. Retrieved from https://www.nichd.nih.gov/ health/topics/preeclampsia/conditioninfo.

2. Lapidus AM. Effects of preeclampsia on the mother, fetus and child; 2010 .

3. Nathan HL, Seed PT, Hezelgrave NL, De Greeff A, Lawley E, Conti-Ramsden $F$, et al. Maternal and perinatal adverse outcomes in women with preeclampsia cared for at facility-level in South Africa: a prospective cohort study. J Global Health. 2018;8(2):020401.

4. National Institute of Population Studies (NIPS)(Pakistan), 2008. Pakistan demographic and health survey 2006-2007.

5. UNICEF. Maternal and Newborn Health Disparities. Pakistan. Retrieved from https://data.unicef.org/wp-content/uploads/country_profiles/Pakistan/ country\%20profile_PAK.pdf.

6. National Institute of Population Studies (NIPS) [Pakistan] and ICF. Pakistan demographic and health survey 2017-18. Islamabad, Pakistan, and Rockville, Maryland, USA: NIPS and ICF; 2018.

7. Mir AM, Pearson S, Shaikh S, Khan M, Masud I, Hussain S. Maternal mortality due to Eclampsia in Khyber Pakhtunkhwa: identifying underlying risk factors, and care-seeking Behaviours. JPMA. 2019;69(7):934-8.

8. Khowaja AR, Qureshi RN, Sheikh S, Zaidi S, Salam R, Sawchuck D, et al. Community's perceptions of pre-eclampsia and eclampsia in Sindh Pakistan: a qualitative study. Reprod Health. 2016;13(1):36.

9. United Nations. Sustainable development goal 3. Ensure healthy lives and promote well-being for all at all ages Retrieved from https:// sustainabledevelopment.un.org/sdg3.
10. Abalos E, Duley L, Steyn DW. Antihypertensive drug therapy for mild to moderate hypertension during pregnancy. Cochrane Database Syst Rev. 2014;2:CD002252.

11. Klocek M, Czarnecka D. Hypertension during pregnancy--how to manage effectively? Przeglad lekarski. 2015;72(4):200-4.

12. Tucker KL, Taylor KS, Crawford C, Hodgkinson JA, Bankhead C, Carver T, et al. Blood pressure self-monitoring in pregnancy: examining feasibility in a prospective cohort study. BMC Pregnancy Childbirth. 2017;17(1):442.

13. Marko Kl, Krapf JM, Meltzer AC, Oh J, Ganju N, Martinez AG, et al. Testing the feasibility of remote patient monitoring in prenatal care using a mobile app and connected devices: a prospective observational trial. JMIR Res Protocols. 2016;5(4):e200.

14. Perry H, Sheehan E, Thilaganathan B, Khalil A. Home blood-pressure monitoring in a hypertensive pregnant population. Ultrasound Obstet Gynecol. 2018;51(4):524-30.

15. Khalii A, Perry H, Lanssens D, Gyselaers W. Telemonitoring for hypertensive disease in pregnancy. Expert Rev Med Devices. 2019;16(8):653-61.

16. Rhoads SJ, Serrano Cl, Lynch CE, Ounpraseuth ST, Gauss CH, Payakachat N, et al. Exploring implementation of $\mathrm{m}$-health monitoring in postpartum women with hypertension. Telemedicine e-Health. 2017;23(10):833-41.

17. Lanssens D, Vandenberk T, Smeets CJ, De Cannière H, Molenberghs G, Van Moerbeke A, et al. Remote monitoring of hypertension diseases in pregnancy: a pilot study. JMIR mHealth and uHealth. 2017;5(3):e25 .

18. Ganapathy R, Grewal A, Castleman J. Remote monitoring of blood pressure to reduce the risk of preeclampsia related complications with an innovative use of mobile technology. Pregnancy Hypertension. 2016;6(4):263-5.

19. Hinton L, Tucker KL, Greenfield SM, Hodgkinson JA, Mackillop L, McCourt C, et al. Blood pressure self-monitoring in pregnancy (BuMP) feasibility study; a qualitative analysis of women's experiences of self-monitoring. BMC Pregnancy Childbirth. 2017;17(1):427.

20. Lanssens D, Vonck S, Storms V, Thijs IM, Grieten L, Gyselaers W. The impact of a remote monitoring program on the prenatal follow-up of women with gestational hypertensive disorders. Eur J Obstet Gynecol Reprod Biol. 2018; 223:72-8.

21. Lanssens D, Vandenberk T, Smeets CJ, De Cannière H, Vonck S, Claessens J, et al. Prenatal remote monitoring of women with gestational hypertensive diseases: cost analysis. J Med Internet Res. 2018;20(3):e102.

22. Xydopoulos G, Perry H, Sheehan E, Thilaganathan B, Fordham R, Khalil A Home blood-pressure monitoring in a hypertensive pregnant population: cost-minimization study. Ultrasound Obstet Gynecol. 2019;53(4):496-502.

23. Monincx $\mathrm{W}$, Zondervan $\mathrm{H}$, Birnie $\mathrm{E}$, Ris $\mathrm{M}$, Bossuyt $\mathrm{P}$. High risk pregnancy monitored antenatally at home. Eur J Obstet Gynecol Reprod Biol. 1997; 75(2):147-53.

24. Buysse H, De Moor G, Van Maele G, Baert E, Thienpont G, Temmerman M. Cost-effectiveness of telemonitoring for high-risk pregnant women. Int $J$ Med Inform. 2008;77(7):470-6.

25. Cairns AE, Tucker KL, Leeson P, Mackillop LH, Santos M, Velardo C, et al. Selfmanagement of postnatal hypertension: the SNAP-HT trial. Hypertension. 2018;72(2):425-32

26. Dalton KJ, Manning K, Robarts PJ, Dripps JH, Currie JR. Computerized home telemetry of maternal blood pressure in hypertensive pregnancy. Int J Biomed Comput. 1987;21(3-4):175-87.

27. Hirshberg A, Vandertuyn M, Mahraj K. Rapid-cycle innovation testing of textbased monitoring for management of postpartum hypertension. J Clin Outcomes Manag. 2017:24:77-85.

28. Martinez B, Ixen EC, Hall-Clifford R, Juarez M, Miller AC, Francis A, et al. mHealth intervention to improve the continuum of maternal and perinatal care in rural Guatemala: a pragmatic, randomized controlled feasibility trial. Reprod Health. 2018;15(1):120.

29. Hirshberg A, Downes K, Srinivas S. Comparing standard office-based followup with text-based remote monitoring in the management of postpartum hypertension: a randomised clinical trial. BMJ Qual Saf. 2018;27(11):871-7.

30. Perry JK, Magann E, McLaughlin B, Chauhan S, Morrison J. Home blood pressure monitoring for pregnant patients with hypertension. J Perinatol. 1998;18(3):226-9.

31. Dunsmuir DT, Payne BA, Cloete G, Petersen CL, Görges M, Lim J, et al. Development of mHealth applications for pre-eclampsia triage. IEEE J Biomed Health Informatics. 2014;18(6):1857-64.

32. Waugh J, Bosio P, Habiba M, Boyce T, Shennan A, Halligan A. Home monitoring of blood pressure in pregnancy at high risk of pre-eclampsia. Eur J Obstet Gynecol Reprod Biol. 2001;99(1):109-11. 
33. Bonnell S, Griggs A, Avila G, Mack J, Bush RA, Vignato J, et al. Community health workers and use of mHealth: improving identification of pregnancy complications and access to care in the Dominican Republic. Health Promot Pract. 2018;19(3):331-40

34. Aquino M, Munce S, Griffith J, Pakosh M, Munnery M, Seto E. Exploring the use of Telemonitoring for patients at high-risk for hypertensive disorders of pregnancy: a scoping review. JMIR Mhealth Uhealth (forthcoming). https:// doi.org/10.2196/15095.

35. Seto E, Leonard KJ, Cafazzo JA, Barnsley J, Masino C, Ross HJ. Perceptions and experiences of heart failure patients and clinicians on the use of mobile phone-based telemonitoring. J Med Internet Res. 2012;14(1):e25.

36. Seto E, Leonard KJ, Cafazzo JA, Barnsley J, Masino C, Ross HJ. Mobile phonebased telemonitoring for heart failure management: a randomized controlled trial. J Med Internet Res. 2012;14(1):e31.

37. Ware P, Ross HJ, Cafazzo JA, Laporte A, Gordon K, Seto E. Evaluating the implementation of a Mobile phone-based Telemonitoring program: longitudinal study guided by the consolidated framework for implementation research. JMIR mHealth and uHealth. 2018;6(7):e10768

38. Ware P, Ross HJ, Cafazzo JA, Laporte A, Seto E. Implementation and evaluation of a smartphone-based telemonitoring program for patients with heart failure: mixed-methods study protocol. JMIR Res Protocols. 2018;7(5): e121.

39. Ware P, Ross HJ, Cafazzo JA, Laporte A, Gordon K, Seto E. User-centered adaptation of an existing heart failure telemonitoring program to ensure sustainability and scalability: qualitative study. JMIR Cardio. 2018;2(2):e11466.

40. Ware P, Dorai M, Ross HJ, Cafazzo JA, Laporte A, Boodoo C, et al. Patient adherence to a Mobile phone-based heart failure Telemonitoring program: a longitudinal mixed-methods study. JMIR mHealth and uHealth. 2019;7(2): e13259.

41. Seto E, Leonard KJ, Cafazzo JA, Barnsley J, Masino C, Ross HJ. Developing healthcare rule-based expert systems: case study of a heart failure telemonitoring system. Int J Med Inform. 2012;81(8):556-65.

42. Seto E, Istepanian RS, Cafazzo JA, Logan A, Sungoor A. UK and Canadian perspectives of the effectiveness of mobile diabetes management systems. 2009 Annual International Conference of the IEEE Engineering in Medicine and Biology Society; 2009: IEEE.

43. Ong SW, Jassal SV, Miller JA, Porter EC, Cafazzo JA, Seto E, et al. Integrating a smartphone-based self-management system into usual care of advanced CKD. Clin J Am Soc Nephrol. 2016;11(6):1054-62.

44. Logan AG, Mclsaac WJ, Tisler A, Irvine MJ, Saunders A, Dunai A, et al. Mobile phone-based remote patient monitoring system for management of hypertension in diabetic patients. Am J Hypertens. 2007;20(9):942-8.

45. Patient Aid foundation. About jinnah postgraduate medical college. Retrieved from https://pafjpmc.org/who-we-are/about-jinnah-postgraduatemedical-college/.

46. National Institute for Health and Care Excellence (NICE) guidleines. Hypertension in pregnancy. Retrieved from https://www.nice.org.uk/ guidance/qs35/chapter/Quality-statement-2-Antenatal-assessment-of-preeclampsia-risk.

47. McBride N. Intervention research: a practical guide for developing evidencebased school prevention programmes: springer; 2016.

48. Braun V, Clarke V. Using thematic analysis in psychology. Qual Res Psychol. 2006;3(2):77-101.

49. Vaismoradi $\mathrm{M}$, Turunen $\mathrm{H}$, Bondas T. Content analysis and thematic analysis: implications for conducting a qualitative descriptive study. Nurs Health Sci. 2013;15(3):398-405.

50. Hsieh H-F, Shannon SE. Three approaches to qualitative content analysis. Qual Health Res. 2005;15(9):1277-88.

51. Ravindran V. Data analysis in qualitative research. Indian J Continuing Nurs Educ. 2019;20(1):40.

\section{Publisher's Note}

Springer Nature remains neutral with regard to jurisdictional claims in published maps and institutional affiliations.

Ready to submit your research? Choose BMC and benefit from:

- fast, convenient online submission

- thorough peer review by experienced researchers in your field

- rapid publication on acceptance

- support for research data, including large and complex data types

- gold Open Access which fosters wider collaboration and increased citations

- maximum visibility for your research: over $100 \mathrm{M}$ website views per year

At BMC, research is always in progress.

Learn more biomedcentral.com/submissions 